\title{
Calculation and comparative analysis of different oilfield water treatment technology
}

\author{
Yang Song ${ }^{\mathrm{a}}$, Qingxin Fan ${ }^{\mathrm{b}}$ \\ School of Municipal \& Environmental Engineering, Harbin Institute of Technology, Harbin, 150096, \\ China. \\ ahitsy@foxmail.com, bfanqingxin@hit.edu.cn
}

Keywords: LCA; Carbon footprint; Sewage treatment plant; GABI model.

\begin{abstract}
As a new measure method that evaluates the influence of carbon emissions, the analysis of carbon footprint can reveal carbon emissions process of different objects from the perspective of life cycle and specifically measure direct and indirect relevant carbon emissions in the whole life cycle of a product or the process of some activity for the purpose of providing the scientific basis for exploring the reasonable and effective ways of greenhouse gas reductions. With the method of life cycle assessment, the paper takes two sewage treatment plants which treat produced water of a certain oilfield in Northeast China as the cases to analyze the use of energy and resources, identify and quantify the carbon emissions in the environment and characterize the list data for the purpose of measuring the carbon emissions of different produced water technologies in the process of construction, operation and abandonment. In this way, it can put forward suggestions for the actual engineering application in the future on the basis of the research results.
\end{abstract}

\section{Introduction}

The latest scientific research shows that the trend of global warming will be accelerated over the next 100 years; it will have more significant negative effects on the natural system and social economy. Therefore, it has become one of the hotspots in the international politics, economy and academic research how to effectively reduce the carbon emissions.

Foreign countries have studied the carbon footprint in the concept connotation, computing method and case measurement, its scale includes individuals, products, families, organizations, cities and countries, and the sectors cover industries, traffic, buildings, water supply and medical institutions. On the whole, it is still in early stage. As a necessary process of daily life and industrial development, wastewater treatment produces muckle carbon emissions during the process of construction, operation and abandonment. However, the study of carbon footprint analysis is rarely focused on wastewater treatment industry.

After more than 70 years of developments, a sound oil-field produced water treatment system has taken shape in an oilfield in Northeast China. Now there are over 200 all forms of sewage treatment stations in Daqing, and the daily wastewater treatment capacity is greater than 2000 thousand tons. At present, most of the oilfields in China have in the middle and later operation periods of petroleum exploitation, the water cut of produced crude oil ranges from $70 \%$ and $80 \%$, even some are greater than $90 \%$. As the water cut increases, the amount of oilfield produced water grows accordingly, so there is more demand for the oilfield produced water treatment. As the different oilfield produced water treatment process brings different carbon footprint, to calculate and analyses the carbon footprint of different oilfield produced water treatment process is expected to be very significant, and can provide reference to the same kind of construction project.

\section{Carbon Footprint Theory}

\subsection{Carbon Footprint}

The concept of "carbon footprint" is based on the ecological footprint, which means the life cycle carbon emissions of the product and also can be said to be the direct and indirect greenhouse (GHG) 
emissions (in $\mathrm{CO}_{2}$ equivalents) of service system or the subject of activity (such as individual, organizations, departments, etc).

Carbon footprint follows the ideology of the whole life cycle, concerns about GHG emissions from the each period in the whole life cycle, such as the raw materials production, processing, translation, distribution, utilization, reutilization, maintaining, and then the waste recycling, final disposal etc. Carbon footprint evaluation contributes to the GHG emissions information tracking and pertinently controls and cuts the GHG emissions.

\subsection{Method of Carbon Footprint Calculation}

Calculating carbon footprint is one of the important and effective ways to evaluate the GHG emissions. At present, carbon footprint calculation can be arrived at mainly by two means: one is the process analysis which is based on the bottom-up model, the other is input-output analysis which is based on the top-down model.

The process analysis method gains input and output data from the life cycle inventory for the basic point of process analyzing. Based on this, the carbon emissions of the whole life cycle can be calculated. The input-output model is the mathematical model used to study the relationship between the input and output of each department in the economic system. As the calculation of carbon dioxide by input-output model is in terms of unit output value, however, oilfield produced water treatment is a public benefit project, the treated water will be injected into ground but not be sold as commodity, this paper choose the process analysis method to calculate the carbon footprint. The process analysis method is based on the life cycle assessment theory, in what follows, the life cycle assessment will be brief introduced.

\subsection{Life Cycle Assessment}

Life cycle assessment (LCA) is a tool that can be used to evaluate the environmental load of a product, process, or activity throughout its life cycle. LCA consists of four parts: goal and scope definition, function unit determining, inventory analysis, and carbon footprints calculations.

Accounting the carbon footprint by LCA, the indeterminacy of method and data should be taken into consideration. Firstly, we should choose applicable method of calculation, including the method of modelling, the treatment of capital goods and land utilization modification, which can have noticeable effect on final outcome. Secondly, the data quality, including accuracy, representative, consistence, reproducibility, data source and the indeterminacy of information, must be up to the standard of ISO14044 and PAS2050.

LCA is a carbon footprint calculating method based on the bottom-up model. The analysis result is pertinent and eligible for carbon footprint calculating of microsystem. Pundits have used this method to account sanitary facilities, biologic energy, miniature communities and economic systems. In the meantime, International Organization for Standardization, British Standard Institution and World Resources Institute have formulated or are formulating the standard of carbon footprint accounting of organizations and products.

\section{Carbon Footprint of Produced Water Treatment Technology}

\subsection{Case Description}

This article will take A sewage treatment plant and B treatment plant of an oilfield in Northeast China as the research objects. They are both located in the First oil production plant, Daqing oilfield. The sewage treatment capacity of the two plants are both $3 \times 104 \mathrm{~m}^{3} / \mathrm{d}$ and the two plants adopt same quality standard for untreated and treated water. The standard is presented in table 1.

Table 1 . The quality standard for untreated and treated oilfield produced water (oilfield waterflood permeability $\mathrm{K}>0.6 \mu \mathrm{m} 2$ )

\begin{tabular}{ccc}
\hline parameter & untreated water & treated water \\
\hline hydraulic pressure & $\geq 0.3 \mathrm{MPa}$ & -- \\
temperature & $\geq 40^{\circ} \mathrm{C}$ & - \\
oil content & $\leq 1000 \mathrm{mg} / \mathrm{L}$ & $\leq 20 \mathrm{mg} / \mathrm{L}$ \\
iron content & -- & $\leq 0.5 \mathrm{mg} / \mathrm{L}$ \\
suspended solids content & -- & $\leq 5 \mathrm{mg} / \mathrm{L}$ \\
\hline
\end{tabular}


A sewage treatment plant adopts the oily wastewater from A dehydration station. The wastewater will be transported into the natural sedimentation tank to remove part of the oil and suspended solid first, then after mixing coagulant through the outlet pipe, the water will be transported into the coagulating sedimentation tank. Lastly, the filtered water will be sent to the Nucleolus Shell Filter to further remove the oil and suspended solid. The effluent water is stored in the clear water tank, and will be transported to the water injection station to recharge the oil-bearing layer of the transmission pump.

B sewage treatment plant adopts the oily wastewater from B dehydration station. The wastewater after mixing coagulant will be transported into the coagulative sedimentation tank first, then mixing flotation chemicals and transported into the flotation machine. Lastly, the filtered water will be sent to the Nucleolus Shell Filter to further remove the oil and suspended solid. The effluent water is will be transported to the water injection station to recharge the oil-bearing layer.

The process flow charts of the two sewage treatment plants are presented in figure 1 and figure 2 .

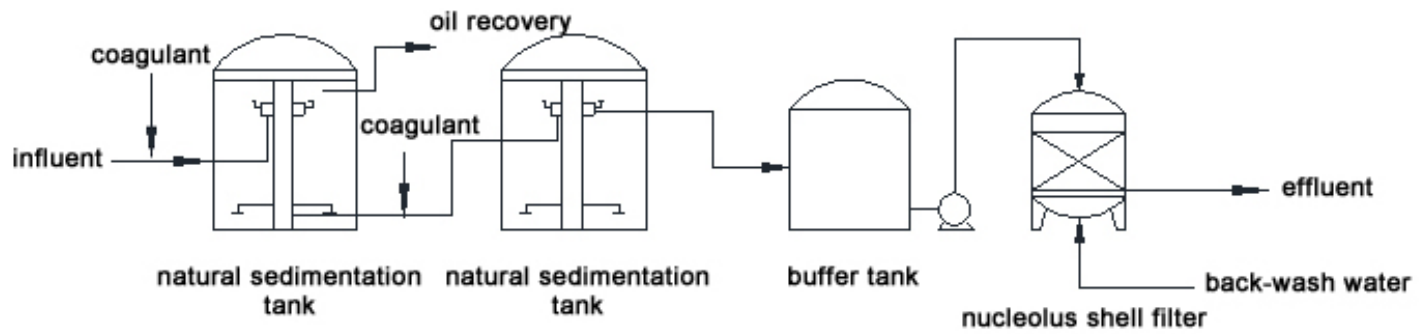

Figure 1. The process flow chart of A sewage treatment plant

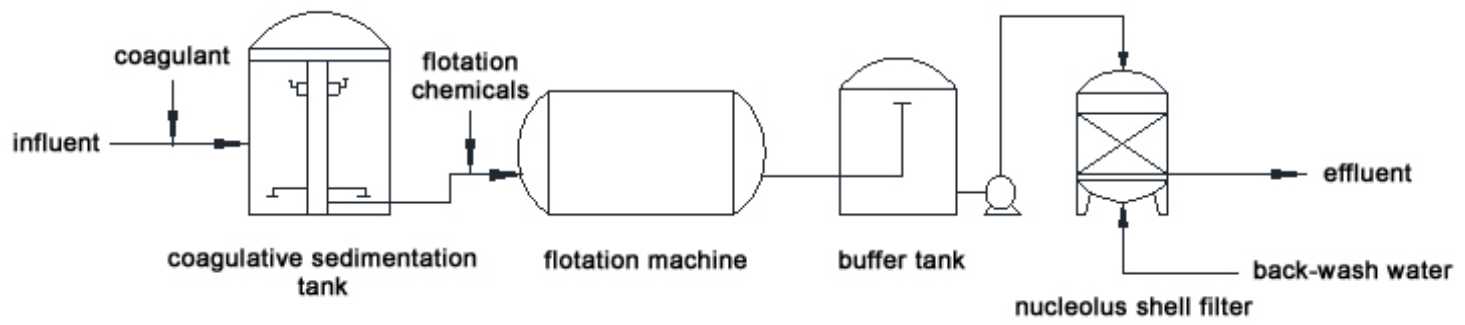

Figure 2. The process flow chart of B sewage treatment plant

\subsection{Border of LCA System}

LCA border in this system starts with the entrance of waste water in the treatment process and ends with the reinjection of standard water after the treatment. The system input includes the consumption of all raw materials and energy in the construction, operation and abandonment stage, and the output includes all of the exhaust gas, waste water and solid waste. The paper does not consider the environmental impact of water, because the standard water from two sewage stations can be injected into the underground rather than the surface water environment after the treatment. The framework of the paper is presented in figure 3. 


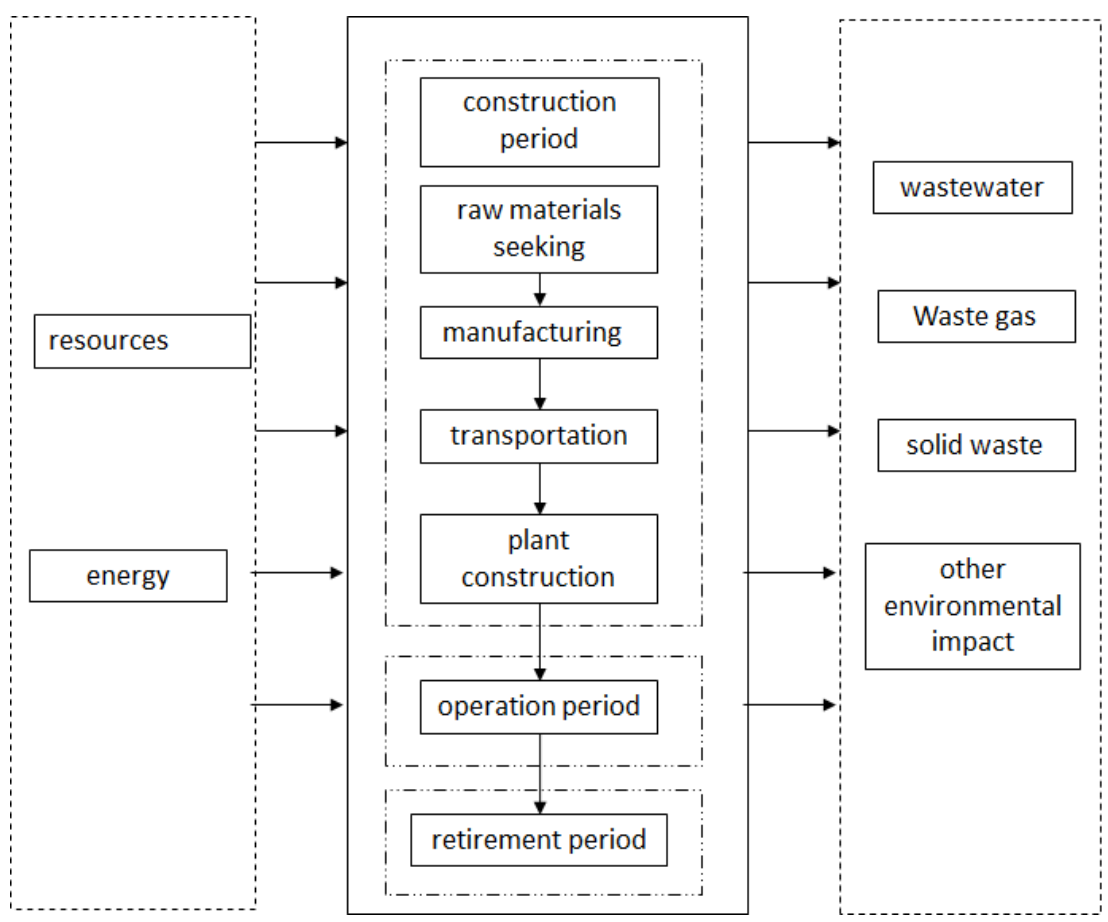

Figure 3. The framework of the LCA system

\subsection{Defining Functional Units}

The purpose of defining functional units is to provide the reference datum for the related input and output data in the sewage treatment system. The paper accounts the whole carbon footprint in the process of construction and abandonment. In the operation period we account the part of carbon footprint from which sewage treatment plant disposes wastewater up to $1.46 \times 10^{7} \mathrm{~m}^{3} / \mathrm{a}$, with annual disposal quantity $1.46 \times 10^{7} \mathrm{~m}^{3} / \mathrm{a}$ as the functional unit.

\subsection{List Analysis of Life Cycle}

As the process that objectively quantifies the data of resources and energy input and environmental release output in the whole sewage treatment process, the list analysis of life cycle is the basis that will affect the assessment in the next step. The non-measurement data of the paper are gained mainly by field investigation, most of which derive from the initial production process descriptions and technology analysis information of factory region.

In the construction phase of sewage treatment plants, the resource consumption mainly includes the steel in production equipment, rebar, cement and other building materials in the related infrastructures. The energy consumption mainly refers to the power consumption in the construction process and the fuel consumption during the transportation of raw materials. The main resource and energy consumption of two sewage treatment plants during the construction stage is presented in table 2 and table 3.

Table 2. The resource and energy consumption of A sewage treatment plant during the construction stage.(electricity: $\mathrm{kw} \cdot \mathrm{h}$, others: $\mathrm{t}$ )

\begin{tabular}{cccc}
\hline item & consumption & item & consumption \\
\hline steel product & 1444.13 & concrete & 436.27 \\
water & 200.64 & electricity & 2391.00 \\
medium(coarse)sand & 587.82 & gravel & 1213.13 \\
spiral welded pipe & 7.34 & welded steel pipe & 0.07 \\
stainless steel pipe & 0.04 & cast iron pipe & 0.24 \\
\hline
\end{tabular}


Table 3. The resource and energy consumption of $\mathrm{B}$ sewage treatment plant during the construction stage (electricity: $\mathrm{kw} \cdot \mathrm{h}$, others: $\mathrm{t}$ )

\begin{tabular}{cccc}
\hline item & consumption & item & consumption \\
\hline steel product & 725.47 & concrete & 219.47 \\
water & 300.73 & electricity & 2009.00 \\
medium(coarse)sand & 610.26 & gravel & 100.93 \\
spiral welded pipe & 7.34 & welded steel pipe & 0.07 \\
stainless steel pipe & 0.04 & cast iron pipe & 0.24 \\
\hline
\end{tabular}

The main energy consumption of the stations is the power consumption in the operation period. The heating in winter is the similar unit in two stations, so the paper does not consider it. Resource consumption is the reagent adding for the equipment operation. The annual resource consumption during the process of operation are presented in table 4.

Table 4. The resource consumption of two sewage treatment plants during the process of operation

\begin{tabular}{ccc}
\hline sewage treatment plant & technology unit & reagent:PAC(kg/d) \\
& natural sedimentation & $/$ \\
& coagulative sedimentation & 1200 \\
& nucleolus shell filter & $/$ \\
B & coagulative sedimentation & 1200 \\
& air floatation & 200 \\
& nucleolus shell filter & $/$ \\
\hline
\end{tabular}

In the abandonment stage, carbon emissions caused by the factory demolition and soil are mainly considered. The floor space related to the technology is smaller in two stations, so they are demolished by the manual and mechanical ways.

Table 5. The resource and energy consumption and the transportation condition of materials during the abandonment process

\begin{tabular}{cccc}
\hline & unit & $\mathrm{A}$ & $\mathrm{B}$ \\
\hline area & $\mathrm{m} 2$ & 1255 & 703 \\
energy consumption & $\mathrm{kw} \cdot \mathrm{h}$ & 119225 & 66785 \\
recycled steel product & $\mathrm{kg}$ & 7711.62 & 8879.78 \\
construction waste & $\mathrm{kg}$ & 1694.25 & 949.05 \\
\hline
\end{tabular}

According to related document statistics, the energy consumption of buildings demolition per unit area is about $95 \mathrm{kwh}$. The building in two stations are reinforced concrete structure, some steel can be recycled in the demolition process, its recovery factor is 0.9 , and others are construction waste. According to the statistics related to the building demolition in China, 1.35 ton of construction waste can be produced when the buildings per square meter are demolished. Above all, the two sewage treatment plants' resource and energy consumption and the transportation condition of materials during the process of abandonment are presented in table 5 .

\subsection{Carbon Footprint Calculation}

The article established the LCA model by means of LCA software GABI, the schematic diagram of which is presented in figure 4. After characterizing the result of list data analysis using the CML2001 characterizing indexes in GABI, we obtained the carbon footprint during the process of construction, operation and abandonment of the two sewage treatment plants, which is presented in table 6 . 


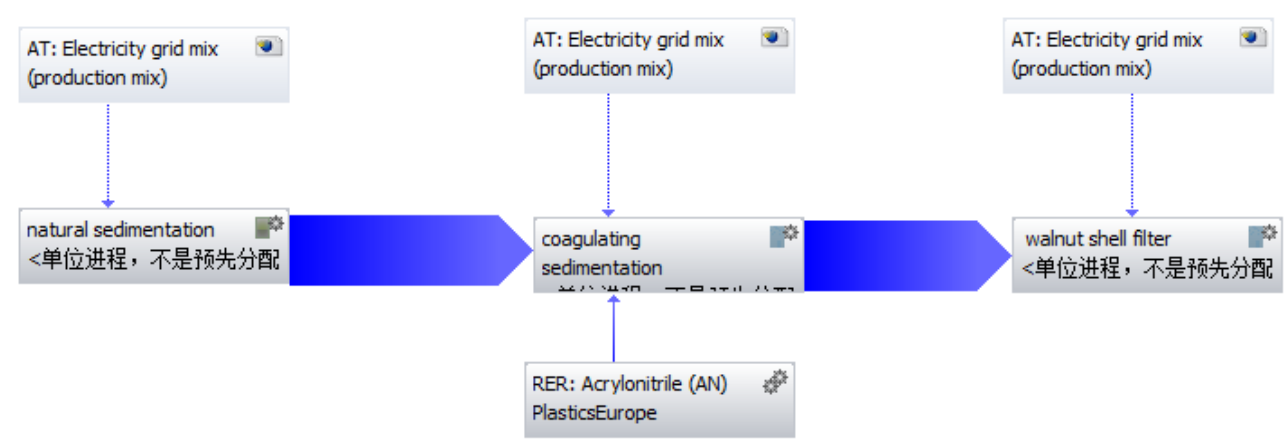

Figure 4. The schematic diagram of GABI model

Table 6. The carbon footprint in different processes of the two sewage treatment plants $(\mathrm{kgCO} 2-$ equiv)

\begin{tabular}{cccc}
\hline & construction process & operation process & abandonment process \\
\hline Middle Fourteen & $5.79 \times 106$ & $3.21 \times 106$ & $2.68 \times 105$ \\
Middle Sixteen & $2.82 \times 106$ & $5.45 \times 106$ & $1.90 \times 105$ \\
\hline
\end{tabular}

\section{Construction Process}

The percentage of each unit's carbon footprint during the construction process is presented in figure 5 .

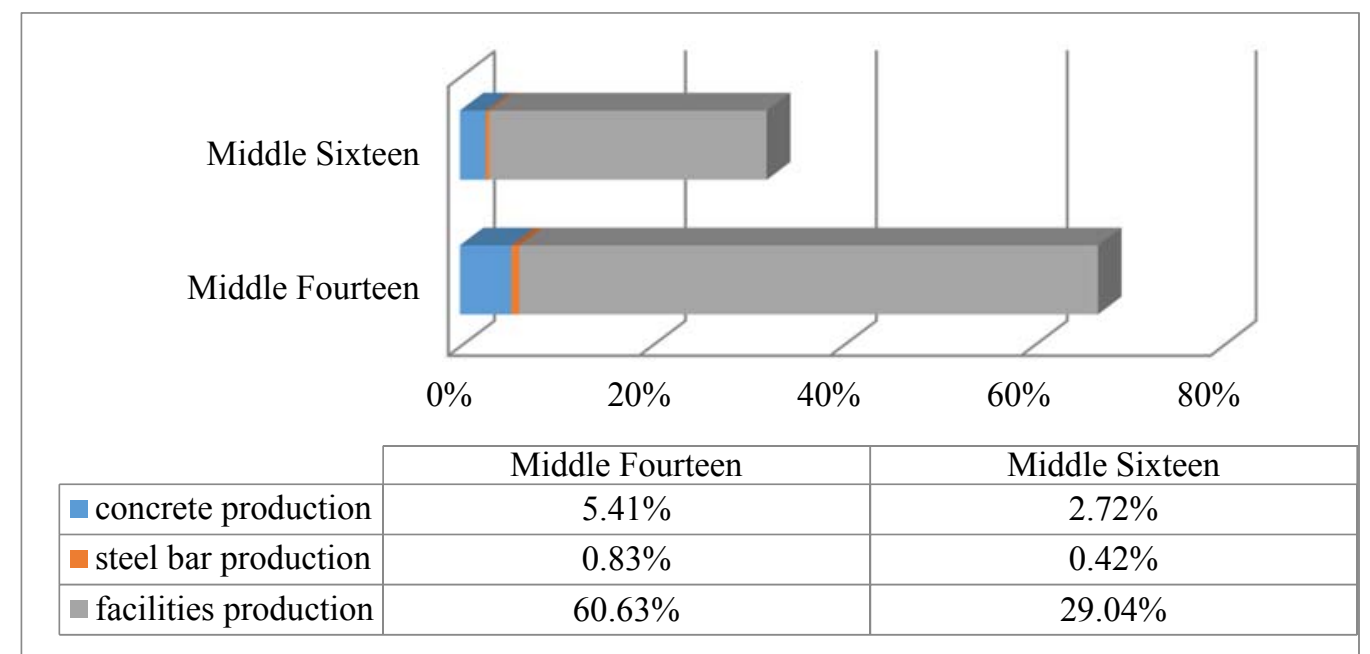

Figure 5. The percentage of each unit's carbon footprint during the construction process

In term of figure 5, the main source of two sewage treatment plants' carbon emission during the construction process is facilities production, about $90 \%$ of the whole carbon emission during the construction. By using natural sedimentation technology, A Sewage Treatment Plant's hydraulic retention time is 4 hours. However, the HRT of B Sewage Treatment Plant which uses air floatation technology is only 0.5 hour. Thus the natural sedimentation tank has the larger volume. The amount of steel bar consumption and facilities production of A Sewage Treatment Plant is 2.09 fold of B's.

\section{Operation process}

The percentage of each unit's carbon footprint during the operation process is presented in figure 6. 


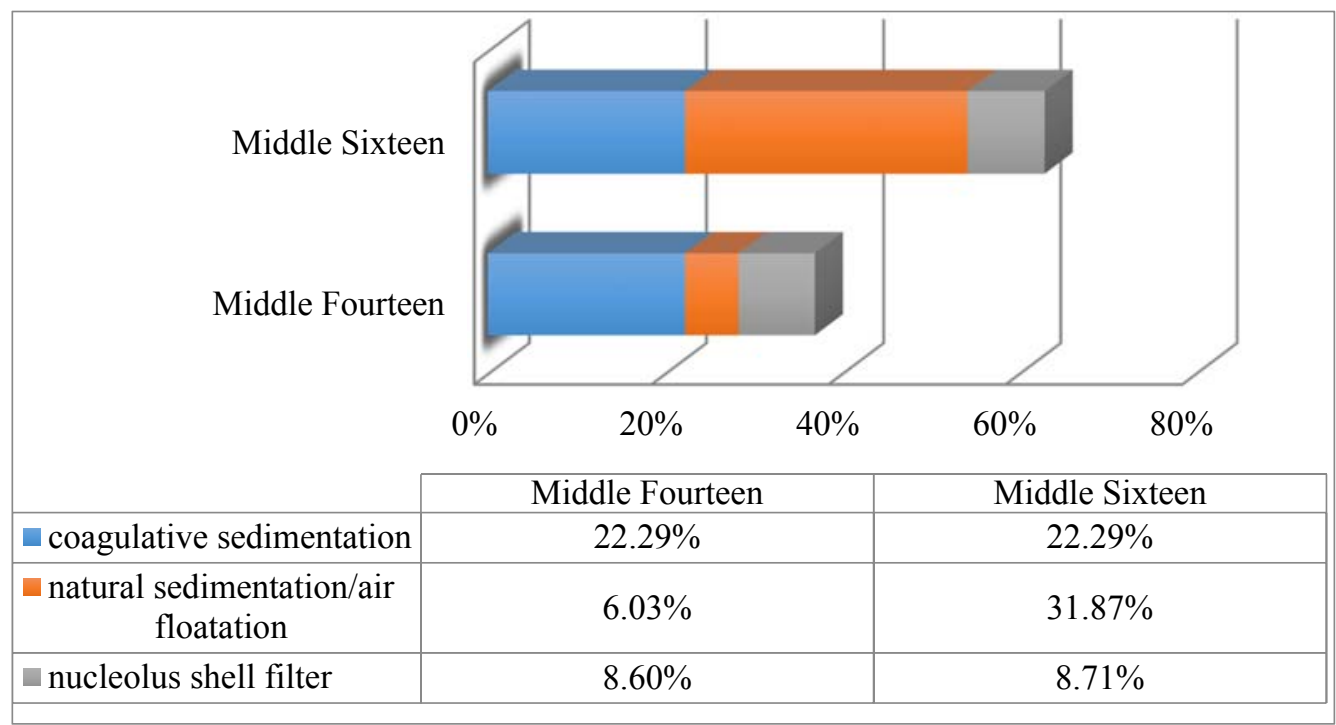

Figure 6. The percentage of each unit's carbon footprint during the operation process

In term of figure 6 , the main source of A sewage treatment plants' carbon emission during the operation process is coagulative sedimentation unit, about $22.29 \%$ of the whole carbon emission during the construction. The main source of carbon emission of B sewage treatment plants during the operation process is air floatation unit, about $31.87 \%$ of the whole, which needs to add flotation agent PCA $400 \mathrm{~kg} / \mathrm{d}$. Moreover, the electricity consumption of air floatation technology is 6.75 fold of natural sedimentation. Thus the amount of operation process carbon footprint of B Sewage Treatment Plant is 1.70 fold of A's.

\section{Abandonment process}

The percentage of each unit's carbon footprint during the abandonment process is presented in figure 7.

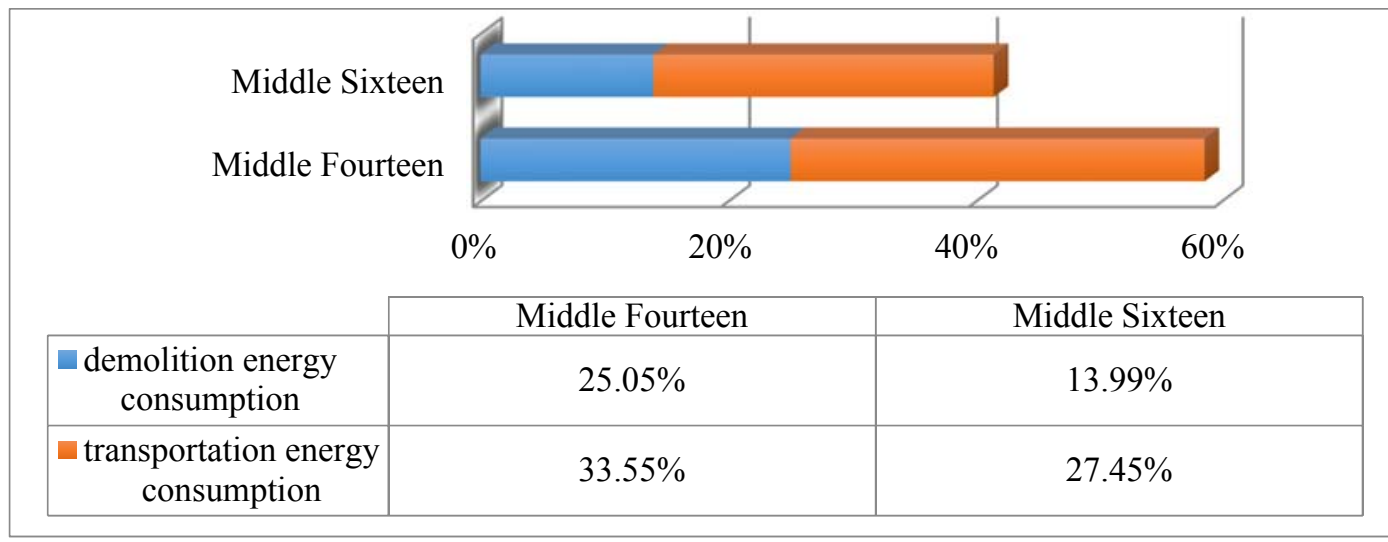

Figure 7. The percentage of each unit's carbon footprint during the abandonment process

According to the table 6, the abandonment process carbon emission makes up a small proportion of the whole life cycle of carbon emission. In term of figure 7, the main source of two sewage treatment plants' carbon emission during the operation process is transportation unit, about $33.44 \%$ and $27.45 \%$ of the whole abandonment process carbon emission.

\section{Sensitivity Analysis}

The purpose of sensitivity analysis is to evaluate the reliability of the results and conclusions by determining whether the results and conclusions are influenced by uncertainties such as data, distribution method or the calculation of type parameters. Usually make alterations of assumption and data range within a certain limit, like $\pm 15 \%$, to examine its influence to the result and then compare the alteration impact. 
Table 7. Main influence contributing units and input materials

\begin{tabular}{ccc}
\hline Plant & Technological units & input materials and unit \\
\hline & facilities production & steel bar(kg) \\
A & natural sedimentation & electricity(MJ) \\
& coagulative sedimentation & electricity(MJ) \\
& nucleolus shell filter unit & PAC(kg) \\
B & facilities production & electricity(MJ) \\
& coagulative sedimentation & steel bar(kg) \\
& air floatation & electricity(MJ) \\
& & PAC(kg) \\
& nucleolus shell filter unit & electricity(MJ) \\
\end{tabular}

According to table 6, in the abandonment process two sewage treatment plants' carbon emission makes up a small proportion of the whole life cycle of carbon emission, $0.23 \%$ and $0.12 \%$ respectively, so the abandonment process is not taken into consideration in sensitivity analysis. The paper chooses four technological processes in the construction and operation period contributing more than 5\% to total environmental impact load, which are facilities production unit, natural sedimentation unit, coagulative sedimentation unit and nucleolus shell filter unit of A plant and facilities production unit, coagulative sedimentation unit, air floatation unit and nucleolus shell filter unit of B plant. Then take related input data as variables to accomplish sensitivity analysis. Related units and input materials are presented in table 7.

According to table 7, after integrating the types of the input materials of different influence contributing units, the paper choose the weight of steel bar, electricity and the weight of PAC as sensitivity analysis variables. By altering $10 \%$ of variables' amount, calculate whole life cycle environmental impact load alteration proportion of A and B wastewater treatment plant, which is presented in table 8 .

Table 8 . The result of sensitivity analysis

\begin{tabular}{ccc}
\hline Variable & $\mathrm{A}$ & $\mathrm{B}$ \\
\hline steel bar(kg) & $3.62 \%$ & $1.49 \%$ \\
electricity $(\mathrm{kw} \cdot \mathrm{h})$ & $4.32 \%$ & $6.90 \%$ \\
PAC $(\mathrm{kg})$ & $6.51 \%$ & $1.35 \%$ \\
\hline
\end{tabular}

According to table 8, alter the three variables of two sewage treatment plants in the same proportion, the whole life cycle environmental impact load alteration proportions caused by the alteration of the same kind of incoming data are quite different, which is due to in the construction and operation process two sewage treatment plants' carbon emission makes different contributions to the whole life cycle of carbon emission. When it comes to Middle Fourteen sewage treatment plant, the alteration of PAC makes up the largest proportion to the whole life cycle of carbon emission, $6.51 \%$. As for Middle Sixteen sewage treatment plant, the alteration of electricity makes up the largest proportion to the whole life cycle of carbon emission, $6.90 \%$.

\section{Results and Discussion}

Take the carbon footprint of the construction and abandonment period of the two sewage treatment plants as initial values, the carbon footprint of operation period as increments; we can get "service time-carbon footprint" function presented in figure 8. 


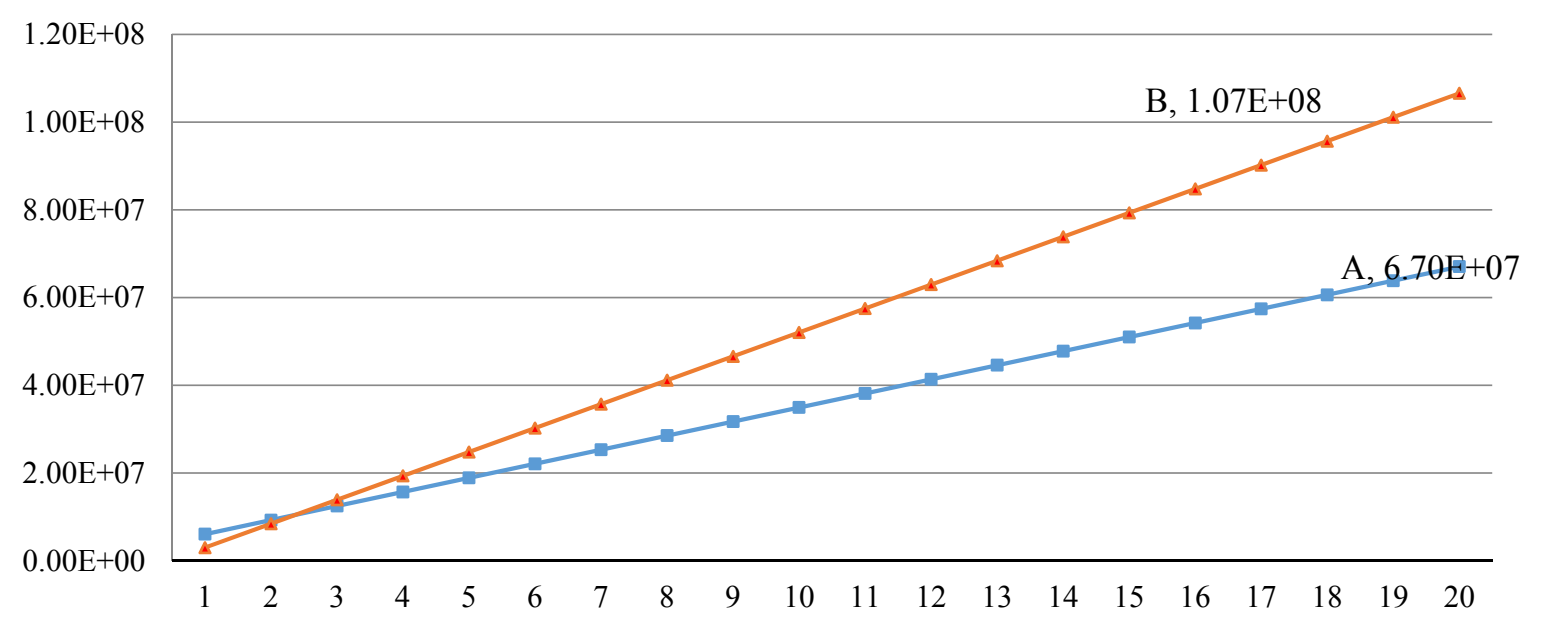

Figure 8. Service time-carbon footprint function

The result of carbon footprint shows that the carbon footprint of the construction and abandonment period of A wastewater treatment plant is higher than B wastewater treatment plant, however when the service time is more than 20 years, the carbon footprint of B wastewater treatment plant would be more than the A wastewater treatment plants. So based on the carbon footprint theory, when the two technology could both meet the treatment demand, the article recommends the process "natural sedimentation-coagulative sedimentation- walnut shell filter".

\section{Conclusion}

The paper takes wastewater treatment plants as a contamination control target, using the evaluation methodology of LCA, calculates the carbon emissions contribution of each unit of wastewater treatment plants during the whole life cycle analyzes the difference of two produced water technologies' carbon footprint and then locates the key factor of influence. Based on the carbon footprint theory, when the two common oilfield water treatment technologies could both meet the treatment demand, the paper recommends the process "natural sedimentation-coagulative sedimentation- walnut shell filter".

\section{References}

[1]. Nobel A. Macro-porous Polymer Extraction for Offshore Produced Water Removes Dissolved and Dispersed Hydrocarbons [J]. Business briefing, exploration \& production, the oil \& gas review, 2004.

[2]. G. Henkelman, G.Johannesson and H. Jónsson, in: Theoretical Methods in Condencsed Phase Chemistry, edited by S.D. Schwartz, volume 5 of Progress in Theoretical Chemistry and Physics, chapter, 10, Kluwer Academic Publishers (2000).

[3]. Allakhverdiev S I, Casal J J, and Nagata T. Photosynthesis from molecular perspectives: towards future energy production [J]. Photochemical \& Photobiological Sciences, 2009,8(2):137-138.

[4]. Cline J T. Treatment and discharge of produced water for deep offshore disposal1998:17-18.

[5]. Woolard C R, Irvine R L. Treatment of hypersaline wastewater in the sequencing batch reactor [J]. Water Research, 1995,29(4):1159-1168.

[6]. Onsuratoom S, Chavadej S, Sreethawong T. Hydrogen production from water splitting under UV light irradiation over Ag-loaded mesoporous-assembled TiO 2-ZrO 2 mixed oxide nanocrystal photocatalysts[J]. international journal of hydrogen energy, 2011,36(9):5246-5261.

[7]. Hudgins Jr C M. Chemical use in North Sea oil and gas E\&P[J]. Journal of Petroleum Technology, 1994,46(01):67-74.

[8]. Thoma G J, Bowen M L, Hollensworth D. Dissolved air precipitation/solvent sublation for oilfield produced water treatment [J]. Separation and Purification Technology, 1999,16(2):101-107. 
[9]. Ebrahimi M, Ashaghi K S, Engel L, et al. Characterization and application of different ceramic membranes for the oil-field produced water treatment [J]. Desalination, 2009,245(1):533-540.

[10]. Mondal S, Wickramasinghe S R. Produced water treatment by nanofiltration and reverse osmosis membranes [J]. Journal of Membrane Science, 2008,322(1):162-170.

[11]. Ebrahimi M, Willershausen D, Ashaghi K S, et al. Investigations on the use of different ceramic membranes for efficient oil-field produced water treatment [J]. Desalination, 2010,250(3):991-996.

[12]. Lardon L, Helias A, Sialve B, et al. Life-cycle assessment of biodiesel production from microalgae[J]. Environmental science \& technology, 2009,43(17):6475-6481.

[13]. Tillman A, Svingby M, Lundström H. Life cycle assessment of municipal waste water systems [J]. The international journal of life cycle assessment, 1998,3(3):145-157.

[14]. Cline J T. Treatment and discharge of produced water for deep offshore disposal1998:17-18.

[15]. Woolard C R, Irvine R L. Treatment of hypersaline wastewater in the sequencing batch reactor [J]. Water Research, 1995,29(4):1159-1168.

[16]. Onsuratoom S, Chavadej S, Sreethawong T. Hydrogen production from water splitting under UV light irradiation over Ag-loaded mesoporous-assembled $\mathrm{TiO} 2-\mathrm{ZrO} 2$ mixed oxide nanocrystal photocatalysts[J]. international journal of hydrogen energy, 2011,36(9):5246-5261.

[17]. Hudgins Jr C M. Chemical use in North Sea oil and gas E\&P[J]. Journal of Petroleum Technology, 1994,46(01):67-74. 